\title{
LEDs \& Lamps - A Friendly Affordable Gateway to Electrical Exploration (Curriculum Exchange)
}

\section{Mr. Andrew Tubesing, University of St. Thomas}

Andrew Tubesing is Laboratory Manager for the Electrical Engineering program at University of St Thomas in St. Paul, MN. He also serves on the faculty of the UST Center for Pre-Collegiate Engineering Education. Andrew has taught university courses in circuits, electronics, and engineering design for more than a decade. Prior to his academic career, Andrew spent 12 years as an engineer in the broadcast and telecommunications fields. Andrew holds a BA from St. Olaf College and a MS in Electrical Engineering from New Mexico Institute of Mining and Technology. 


\title{
LEDs \& Lamps - A Friendly \& Affordable Gateway to Electrical Exploration ASEE 2014 Conference - K-12 Division - Curriculum Exchange \\ Andrew Tubesing, University of St Thomas
}

\begin{abstract}
Light emitting diodes (LEDs) and incandescent lamps can be used to explore electrical topics, substitute for otherwise expensive equipment, facilitate creative engineering design projects, and provide opportunities to integrate math, science, engineering and art with real-world issues such as energy efficiency and technology for the developing world. This presentation aims to empower teachers to further explore electrical topics, despite whatever limitations they may feel in terms of funds, resources, and/or technical knowledge. A variety of ideas for various age ranges, together spanning the full K-12 spectrum, are covered.
\end{abstract}

\section{Pedagogical Context}

LEDs and lamps are popular and fun components that help make learning electrical topics a playful experience. This demonstration explores their many appealing applications for STEM education...

1. LEDs and lamps can be used to observe both static and dynamic circuit behavior, offering a visual method of learning about electricity and providing a substitute for expensive measurement instruments. LED brightness corresponds to current, incandescent lamp brightness corresponds to voltage. These fundamental electrical concepts are made tangible and accessible by observing them with light.

2. LEDs and lamps make for great hands-on projects that develop practical skills (such as schematic diagram interpretation, wiring dexterity, soldering, and circuit analysis) and offer compelling creative design opportunities - both technical and aesthetic.

3. LEDs and lamps are commonly used in energy efficiency improvements and products designed to make best use of limited resources in the developing world. This offers an opportunity to integrate engineering within the framework of social/world issues while including varying levels of scientific content as needed.

\section{Demonstrations}

The reverse side of this sheet summarizes a selection of hands-on light-based circuit activities that demonstrate and help teach electrical concepts. The 'measurements' can all be performed by visual observation without instruments.

This presentation showcases these and/or other similar experiments, which are listed in order of target age with annotations for various math integration opportunities (not requirements). All are compatible with physical science and/or physics at any level.

\section{Presenter information}

Feel free to contact the presenter for help or ideas in applying these topics in your classroom or lab.

Andrew Tubesing

Electrical Engineering Laboratory Manager

University of St. Thomas - School of Engineering \& Center for Engineering Education

2115 Summit Ave - Mailstop OSS100 - St Paul MN 55105-1079

(651) 962-5437 Tel - (651) 962-6419 Fax

tubesing@stthomas.edu

http://www.stthomas.edu/engineering/faculty/adTubesing.htm 
1. LEDs and energy efficiency (Grades: K-12, Math: multiplication and beyond): A simple battery-operated LED torch can be compared to a traditional incandescent night light by calculating the power usage of each light source. The LED version uses $1 / 25$ as much power as the incandescent night light. Additional lessons can be explored regarding the design process, including balancing brightness versus power usage and battery life, and the benefits and opportunities that LEDs bring to our world. This is a very effective introductory project that is appropriate for any age (beyond choking hazard).

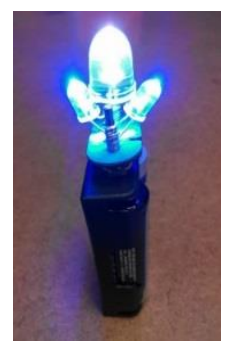

2. Potentiometer as voltage/current control (Grades: 4-12, Math: addition \& subtraction): A lamp and LED are be wired such that they get dimmer and brighter as the potentiometer is adjusted. The lamp and LED brightness indicate voltage and current, respectively. The concept of voltage division can also be explored by using a circuit with two LEDs.

3. Resistor as a current limiter and LED as current indicator (Grades: 5-12, Math: addition, subtraction, multiplication): Several series (connected end-to-end) resistor/LED combinations are wired in parallel (connected to each other at both ends). Each combination will have an identical LED and different size resistor. LED brightness indicates current, thus enabling visual confirmation of current division by observing larger/smaller currents in the circuit which correspond to smaller/larger resistances.

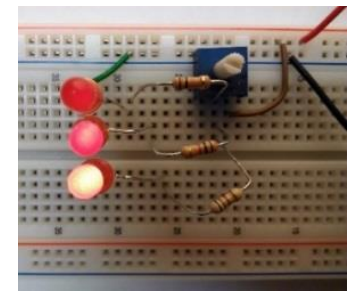

4. Logic Probe (Grades: 6-12, Math: binary): Simple circuit with 2 LEDs and 2 resistors to indicate $\mathrm{Hi} / \mathrm{LO}$ status of a digital circuit pin. This is useful to test/indicate the status (HI or LO) of any node in a digital circuit. Applications include anything from digital logic circuits to microprocesser-based systems such as arduino, beagle, and raspberry pi boards, among many others.

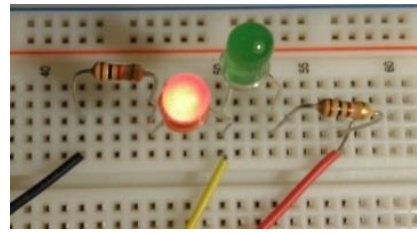

5. Alternating current (Grades: 6-12, Math: sine waves \& trigonometry): A bipolar LED (or pair of opposite-wired different-colored LEDs in parallel) are connected to an AC sine wave signal. One color lights when the positive peaks pass and the other lights when the negative peaks pass, indicating the forward/reverse current behavior of an AC system and the one-way current properties of a diode.

6. Rectifier/AC-DC converter (Grades: 7-12, Math: sine waves \& trigonometry): A single LED wired to an AC sine wave demonstrates the half-wave rectifier concept (the diode only allows the positive peaks to pass). A four-LED full-wave rectifier illuminates the current flow as it cleverly inverts the negative peaks. An incandescent lamp wired to the output of both (indicating the output voltage) demonstrates that the full-wave rectifier generates a smoother output. A capacitor can be added to each rectifier such that the lamp also shows the

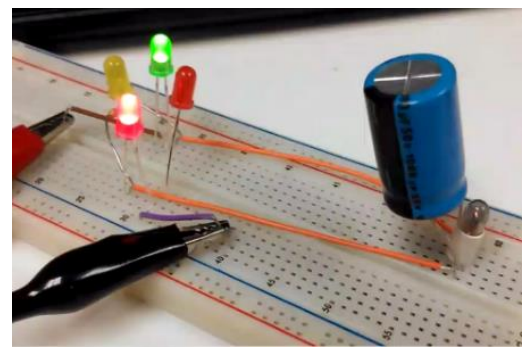
capacitor's additional smoothing affect.

7. Capacitor charge/discharge (Grades 10-12, Math: Pre-calc \& Calculus): A capacitor circuit is wired to a pulse source that cycles on and off such that the cap charges and discharges repeatedly. An incandescent lamp wired in parallel with the cap indicates its voltage, growing brighter as the cap charges and dimmer as it discharges. An LED wired in series with the cap indicates its current, which is the rate of change of voltage; it will light only when the voltage is changing (charging or discharging), and turn off when the cap is fully charged or discharged (when there is no voltage change). The derivative relationship between capacitor voltage and current can be observed dynamically in the brightness of the lamp and LED.

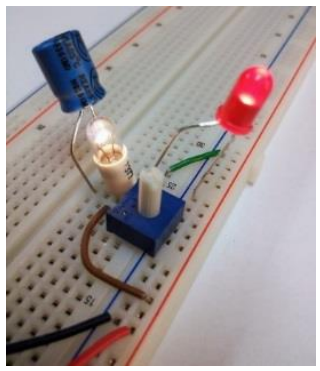

